\title{
AN ARITHMETIC PROPERTY OF RIEMANN SUMS ${ }^{1}$
}

\section{WALTER RUDIN}

If $f$ is a real function on the real line, periodic with period 1 , define

$$
\left(M_{n} f\right)(x)=\frac{1}{n} \sum_{i=1}^{n} f\left(x+\frac{i}{n}\right) \quad(n=1,2,3, \cdots) .
$$

Writing $\int f$ for the integral of $f$ over $[0,1]$, the relation

$$
\lim _{n \rightarrow \infty}\left(M_{n} f\right)(x)=\int f
$$

holds for all real $x$ if $f$ is Riemann integrable on $[0,1]$. In the present note it is shown that there are bounded measurable functions $f$ for which (2) is false for every $x$ and that this convergence problem has some interesting number-theoretic aspects.

In 1934, Jessen [1] proved that if $f \in L^{1}$ on $[0,1]$ and if $\left\{n_{k}\right\}$ is an increasing sequence of positive integers in which each term divides the next, then

$$
\lim _{k \rightarrow \infty}\left(M_{n_{k}} f\right)(x)=\int f
$$

for almost all $x$.

In 1948, Salem [2] showed that (3) holds for almost all $x$ if the integral modulus of continuity of $f$ satisfies a certain condition and if $\left\{n_{k}\right\}$ satisfies a corresponding lacunarity condition. Salem's condition involves only the rate of growth of $\left\{n_{k}\right\}$; no divisibility assumptions appear.

In the opposite direction, it is known (I am indebted to the referee for mentioning [4] and [5]) that there are functions $f \in L^{1}$ for which

(2) fails almost everywhere. For example, if $0<\alpha<1 / 2$, define

$$
f(x)=|x|^{-1+\alpha} \quad(|x| \leqq 1 / 2)
$$

and define $f(x)$ for all other $x$ by periodicity. For every irrational $x$, there are infinitely many integers $n$ such that

$$
\left|x-\frac{m}{n}\right|<\frac{1}{n^{2}}
$$

Presented to the Society, February 25, 1963, under the title Prime numbers and measurable functions; received by the editors February 8, 1963.

1 This research was supported by NSF grant GP-249. 
for some integer $m$; if (5) holds, then $f(x-m / n)>f\left(1 / n^{2}\right)=n^{2-2 \alpha}$ so that $\left(M_{n} f\right)(x)>n^{1-2 \alpha}$. Thus

$$
\limsup _{n \rightarrow \infty}\left(M_{n} f\right)(x)=+\infty
$$

for almost all $x$. If $p<2$, we may choose $\alpha$ so that $1-1 / p<\alpha<1 / 2$, and thus get examples of $f \in L^{p}$ for which (6) holds almost everywhere.

This crude method does not settle the problem for $L^{2}$, nor, a fortiori, for bounded measurable functions. However, (2) fails even there, and it turns out that arithmetic properties of $\left\{n_{k}\right\}$ are crucial; Remark (A) at the end of this note makes this very evident.

THEOREM. Let $S$ be a sequence of positive integers which contains sets $S_{N}(N=1,2,3, \cdots)$, each consisting of $N$ terms, such that no member of $S_{N}$ divides the least common multiple of the other members of $S_{N}$.

Then to every $\epsilon>0$ there exists a bounded measurable function $f$, periodic with period 1 , such that $0 \leqq f \leqq 1$, and such that

$$
\lim _{n \in S} \sup \left(M_{n} f\right)(x) \geqq \frac{1}{2}
$$

for all $x$, although $\int f<\epsilon$.

For instance, $S$ could be any sequence of primes.

In the proof, $f$ is constructed as the characteristic function of an open set.

Proof. We may assume, without loss of generality, that the sets $S_{N}$ are pairwise disjoint.

Fix $N>2$, choose $\delta>0$ such that $\delta^{N}=N^{-1}(\log N)^{-2}$, let $g$ and $h$ be the characteristic functions of sets $G$ and $H$, where $G$ is the union of the segments $(k, k+\delta)(k=0, \pm 1, \pm 2, \cdots)$, and $H$ is the complement of $G$. If $n_{1}, \cdots, n_{N}$ are the members of $S_{N}$, let $k_{i}$ be the least common multiple of $n_{1}, \cdots, n_{i-1}, n_{i+1}, \cdots, n_{N}$, for $i=1, \cdots, N$. We can then find integers $p_{1}, \cdots, p_{N}$, each so much larger than the preceding one that the following is true: if

$$
\begin{aligned}
\phi_{N}(t) & =\prod_{i=1}^{N} g\left(k_{i} p_{i} t\right), \\
\psi_{j, N}(t) & =h\left(k_{j} p_{j} t\right) \prod_{i \neq j} g\left(k_{i} p_{i} t\right) \quad(1 \leqq j \leqq N),
\end{aligned}
$$

then $\int \phi_{N}$ and $\int \psi_{j, N}$ differ by as little as we please from the products

$$
\left(\int g\right)^{N}=\delta^{N}
$$

and 


$$
\left(\int h\right)\left(\int g\right)^{N-1}=(1-\delta) \delta^{N-1}
$$

Put $A_{N}=B_{1, N} \cup \ldots \cup B_{N, N}$, where $B_{j, N}$ is the set whose characteristic function is $\psi_{j, N}$. Since the sets $B_{1, N}, \cdots, B_{N, N}$ are pairwise disjoint, and since

$$
N(1-\delta) \delta^{N-1}=N \delta^{N}\left(\frac{1}{\delta}-1\right)>N \delta^{N}\left(N^{1 / N}-1\right)>\delta^{N} \log N,
$$

we see from $(7)$ and $(8)$ that $p_{1}, \cdots, p_{N}$ can be so chosen that

$$
\int \phi_{N}<\frac{2}{N \log ^{2} N}, \quad m\left(A_{N}\right)>\frac{1}{N \log N},
$$

where $m\left(A_{N}\right)$ denotes the Lebesgue measure of $A_{N} \cap[0,1]$. Moreover, $p_{1}, \cdots, p_{N}$ can be chosen to be primes which divide no member of $S_{N}$.

Suppose now that $x \in B_{j, N}$. Then $k_{i} p_{i} x \in G$ if $i \neq j$, and $k_{j} p_{j} x \in H$. Since $n_{j}$ divides $k_{i}$ if $i \neq j$, we have

$$
k_{i} p_{i}\left(x+\frac{r}{n_{j}}\right) \equiv k_{i} p_{i} x(\bmod 1)
$$

if $i \neq j$ and $r=1, \cdots, n_{j}$. But $n_{j}$ does not divide $k_{j} p_{j}$ (this is where the arithmetic hypothesis imposed on $S_{N}$ is used), and therefore the terms of the arithmetic progression

$$
k_{j} p_{j}\left(x+\frac{r}{n_{j}}\right) \quad\left(r=1, \cdots, n_{j}\right)
$$

are not all congruent $(\bmod 1)$; since $\delta>1 / 2$, it follows that at least half of them lie in $G$. Hence

$$
\left(M_{n_{j}} \phi_{N}\right)(x) \geqq \frac{1}{2}
$$

for all $x \in B_{j, N}$, and (12) implies that

$$
\max _{n \in S_{N}}\left(M_{n} \phi_{N}\right)(x) \geqq \frac{1}{2} \quad\left(x \in A_{N}\right) .
$$

By (9), $\sum m\left(A_{N}\right)=\infty$. Hence there is a sequence $\left\{\alpha_{N}\right\}$ of real numbers such that almost every $x$ lies in infinitely many of the translates $A_{N}+\alpha_{N}$ (see $\left[3\right.$, p. 165 , Lemma 1.24]). Choose $N_{0}$ so that

$$
4 \sum_{N_{0}}^{\infty} N^{-1}(\log N)^{-2}<\epsilon
$$

and put 


$$
\phi(t)=\sup _{N \geqq N_{0}} \phi_{N}\left(t-\alpha_{N}\right) .
$$

Then (13) implies

$$
\lim _{n \in S} \sup \left(M_{n} \phi\right)(x) \geqq \frac{1}{2}
$$

for almost all $x$, although $\int \phi<\epsilon / 2$, by (9) and (14).

If now $E$ is the set of measure 0 on which (16) fails, let $\chi$ be the characteristic function of a periodic open set $V$ which contains $E+r$ for all rational numbers $r$, and such that $m(V)<\epsilon / 2$. Setting $f=\max (\phi, \chi)$, we obtain a function which has the properties asserted by the theorem.

REMARKs. (A) There are sequences $\left\{n_{k}\right\}$ which satisfy the hypothesis of Jessen's theorem but such that $\left\{1+n_{k}\right\}$ is a sequence of primes. To see this, suppose $n_{k}$ is chosen; by Dirichlet's theorem on primes in arithmetic progressions, there is an integer $r>1$ such that $q=1+r n_{k}$ is prime; put $n_{k+1}=r n_{k}$.

Thus $\left\{\left(M_{n_{k}} f\right)(x)\right\}$ converges to $\int f$ a.e. although the sequence $\left\{\left(M_{1+n k} f\right)(x)\right\}$ need not do so.

(B) Take $\epsilon_{k}=2^{-k}$ in our theorem, let $f_{k}(f=1,2,3, \cdots)$ be the corresponding functions, and put $F=\sum k f_{k}$. Then $F \in L^{p}$ on $[0,1]$ for every $p<\infty$, but

$$
\limsup _{n \in S}\left(M_{n} F\right)(x)=+\infty
$$

for every $x$.

(C) It is easy to see that $M_{n} f \rightarrow f$ in the $L^{p}$-norm, as $n \rightarrow \infty$, for every $f \in L^{p}$, if $1 \leqq p<\infty$. Hence for every $f \in L^{1}$ there is a sequence $\left\{n_{k}\right\}$ such that (3) holds almost everywhere.

\section{REFERENCES}

1. B. Jessen, On the approximation of Lebesgue integrals by Riemann sums, Ann. of Math. (2) 35 (1934), 248-251.

2. R. Salem, Sur les sommes Riemanniennes des fonctions sommables, Mat. Tidsskr. B 1948, 60-62 (1948).

3. A. Zygmund, Trigonometric series, 2nd ed., Vol. II, Cambridge Univ. Press, Cambridge, 1959.

4. J. Marcinkiewicz and A. Zygmund, Mean values of trigonometrical polynomials, Fund. Math. 28 (1937), 131-166.

5. H. D. Ursell, On the behaviour of a certain sequence of functions derived from a given one, J. London Math. Soc. 12 (1937), 229-232.

UNIVERSITY OF WISCONSIN 